Apidologie, 1980, 11 (1), 25-28.

\title{
DIE ELEKTRISCHE LEITFÄHIGKEIT VON HONIGEN AUS SANTIAGO DEL ESTERO, ARGENTINIEN
}

\section{La conductibilité électrique des miels de la province de Santiago del Estero en Argentine}

\author{
Eduardo Mario BIANCHI \\ Centro de Investigaciones Apicolas C.E.D.I.A. \\ Universidad Nacional de Santiago del Estero \\ Av. Moreno (S) 577 4.200 Santiago del Estero \\ (Rep. Argentina).
}

\section{SUMMARY}

ELECTRIC CONDUCTIBILITY OF HONEYS IN SANTIAGO DEL ESTERO, ARGENTINA

36 honey samples were collected in the province of Santiago del Estero in northern Argentina. $20 \%$ honey solution (based on dry matter) at a temperature of $20^{\circ} \mathrm{C}$ was used for the measurements. The samples show conductivity values ranging between $1.73-13.80 \cdot 10^{-4} \mathrm{~S} \cdot \mathrm{cm}^{-1}$. The same range of values was found in European honeys.

\section{ZUSAMMENFASSUNG}

In der Provinz Santiago des Estero, die im Norden Argentiniens gelegen ist, wurden 36 Honigproben gesammelt. Die Messungen erfolgten in $20 \%$ Lösungen (bezogen auf das Trockengewicht) bei $20^{\circ} \mathrm{C}$. Die Werte der spezifischen elektrischen Leitfähigkeit schwanken zwischen $1.73-13.8 \cdot 10^{-4} \mathrm{~S} \cdot \mathrm{cm}^{-1}$. Das entspricht der Variationsbreite, die bei Messungen mit europäischen Honigen beobachtet wurde.

\section{EINLEITUNG}

Unter elektrischer Leitfähigkeit versteht man das Vermögen einer Lösung, den elektrischen Stron zu leiten. Die Lösungen folgen wie metallische Leiter dem 
Ohm'schen Gesetz, abgesehen von extremen Bedingungen wie z.B. hohen Spannungen oder hohen Frequenzen.

Die Masseinheit der spezifischen Leitfähigkeit $\varkappa(K a p p a)$ ist der reziproke Wert des Ohm'schen Widerstands eines Flüssigkeitskubus von $1 \mathrm{~cm}$ Seitenlänge bei einer bestimmten Temperatur. Die Messwerte werden in $\mathrm{Ohm}^{-1} \cdot \mathrm{cm}^{-1}$ angegeben oder in $\mathrm{S} \cdot \mathrm{cm}^{-1}$, wobei $\mathrm{S}$ für Siemens steht $\left(\mathrm{S}=\mathrm{Ohm}^{-1}\right)$. ElSER (1924) wies als erster auf die diagnostische Bedeutung der elektrischen Leitfähigkeit des Honigs hin. Später taten es auch Stitz u. SzigvarT und zuletzt Vorwohl (1964 a, 1964 b).

\section{MATERIAL UND METHODEN}

Die Provinz Santiago del Estero liegt im Norden Argentiniens zwischen dem 28. und 30. Breitengrad der Südhalbkugel, also im subtropischen Bereich. Der grösste Teil der Provinz gehört noch zu der ausgedehnten Argentinischen Tiefebene. Im Osten reicht sie bis zu den Vorbergen der Anden. Das Gebiet umfasst Savannen und Steppen und bewässertes Kulturland. 36 Honigproben wurden freundlicherweise von den Bienenzüchtern zur Verfügung gestellt. Die Orte, an denen sie erhoben wurden, sind der Tabelle 1 $\mathrm{zu}$ entnehmen.

Bei den Messungen verfuhren wir, wie bei VORWOHL (1964 a) beschrieben.

\section{ERGEBNISSE UND DISKUSSION}

Die Tabelle 1 bringt eine Zusammenstellung der Messwerte. Sie bewegen sich zwischen 1.78 und $13.80 \cdot 10^{-4} \mathrm{~S} \cdot \mathrm{cm}^{-1}$. Die Leitfähigkeit zeigt demnach eine beachtliche Variabilität. Auf Grund der Farbe des Honigs kann die Leitfähigkeit annähernd vorausgesagt werden. Je dunkler der Honig, desto grösser die Leitfähigkeit.

Entsprechend bestehen Beziehungen zur trachtmässigen Herkunft der Honige. Die technischen Voraussetzungen zu einer genaueren mikroskopischen Sortendiagnose fehlen momentan noch. Ausgesprochene Sortenhonige, deren Diagnose der Sinnenbefunde ausreicht, sind in Santiago del Estero selten.

Aufgrund der bisherigen Literaturergebnisse kann man davon ausgehen, dass die meisten untersuchten Honige Blütenhonige sind. 3 Honigproben zeigen spez. Leitfähigkeiten über $10 \cdot 10^{-4} \mathrm{~S} \cdot \mathrm{cm}^{-1}$ und dürften demnach Honigtauhonige sein. Bei den Proben mit Leitfähigkeiten über $6 \cdot 10^{-4} \mathrm{~S} \cdot \mathrm{cm}^{-1}$ sind Honigtauanteile zu vermuten, da Blütenhonige mit Leitfähigkeiten von mehr als $6 \cdot 10^{-4} \mathrm{~S} \cdot \mathrm{cm}^{-1}$ selten sind (z.B. Myosotis silvatica 6.79 und Echinops commutatus $8.47 \cdot 10^{-4} \mathrm{~S} \cdot \mathrm{cm}^{-1}$ ).

Die Variabilität der Leitfähigkeit entspricht genau der Spanne, die bei europäischen Honigen zwischen hellen Blütenhonigen und ausgesprochenen Honigtauhonigen gefunden wurde. In dieser Beziehung scheinen also für Südamerika die gleichen Gesetzmässigkeiten zu gelten. 
ТАв. 1. - Spezifische elektrische Leitfähigkeit der untersuchten Honige

TABL. 1. - Conductibilité électrique spécifique des miels étudiés

\begin{tabular}{|c|c|c|c|c|c|}
\hline \multirow{2}{*}{$\begin{array}{c}\text { Probe } n^{\circ} \\
\text { Échantillon } n^{\circ}\end{array}$} & \multicolumn{2}{|c|}{$\begin{array}{l}\text { Ernte } \\
\text { Récolte }\end{array}$} & \multicolumn{2}{|c|}{$\begin{array}{c}\text { Herkunft } \\
\text { Origine }\end{array}$} & \multirow{2}{*}{$\begin{array}{l}\text { Elektrische Leitfähigkeit } \\
\mathrm{Z}\left(10^{-4} \cdot \mathrm{ohm}^{-1} \cdot \mathrm{cm}^{-1}\right) \\
\text { Conductibilité électrique } \\
\mathrm{Z}\left(10^{4} \cdot \mathrm{ohm}^{-1} \cdot \mathrm{cm}^{1}\right)\end{array}$} \\
\hline & $\begin{array}{c}\text { Jahr } \\
\text { Année }\end{array}$ & $\begin{array}{c}\text { Monat } \\
\text { Mois }\end{array}$ & $\begin{array}{l}\text { Ort } \\
\text { Lieu }\end{array}$ & $\begin{array}{l}\text { Bezirk } \\
\text { District }\end{array}$ & \\
\hline 313 & 1978 & Febr. & Selva & Rivadavia & 1,78 \\
\hline 275 & 1977 & Nov. & Huaycurú & Banda & 2,27 \\
\hline $\begin{array}{l}272 \\
273 \\
271 \\
274 \\
279 \\
302 \\
318 \\
277\end{array}$ & $\begin{array}{l}1977 \\
1977 \\
1977 \\
1977 \\
1977 \\
1978 \\
1977 \\
1977\end{array}$ & $\begin{array}{l}\text { Nov. } \\
\text { Nov. } \\
\text { Nov. } \\
\text { Oktob. } \\
\text { Dez. } \\
\text { Febr. } \\
\text { Oktob. } \\
\text { Oktob. }\end{array}$ & $\begin{array}{l}\text { La Abrita } \\
\text { La Aurora } \\
\text { Simbol } \\
\text { Nva. Francia } \\
\text { Sta. Maria } \\
\text { San Ramón } \\
\text { Yanda } \\
\text { Beltrán }\end{array}$ & $\begin{array}{l}\text { Silipica } \\
\text { Banda } \\
\text { Silipica } \\
\text { Silipica } \\
\text { Hauptbezirk } \\
\text { Banda } \\
\text { Hauptbezirk } \\
\text { Banda }\end{array}$ & $\begin{array}{l}3,19 \\
3,21 \\
3,37 \\
3,41 \\
3,59 \\
3,62 \\
3,87 \\
3,98\end{array}$ \\
\hline $\begin{array}{l}301 \\
315 \\
312 \\
278 \\
276 \\
332 \\
308\end{array}$ & $\begin{array}{l}1977 \\
1978 \\
1977 \\
1977 \\
1977 \\
1978 \\
1978\end{array}$ & $\begin{array}{l}\text { Nov. } \\
\text { Febr. } \\
\text { Dez. } \\
\text { Nov. } \\
\text { Oktob. } \\
\text { Jan. } \\
\text { Jan. }\end{array}$ & $\begin{array}{l}\text { Est. Zanjón } \\
\text { Clodomira } \\
\text { Ardiles } \\
\text { Vinalar } \\
\text { Maco } \\
\text { La Punta } \\
\text { Forres }\end{array}$ & $\begin{array}{l}\text { Hauptbezirk } \\
\text { Banda } \\
\text { Banda } \\
\text { Hauptbezirk } \\
\text { Hauptbezirk } \\
\text { Choya } \\
\text { Robles }\end{array}$ & $\begin{array}{l}4,10 \\
4,12 \\
4,39 \\
4,48 \\
4,59 \\
4,71 \\
4,97\end{array}$ \\
\hline $\begin{array}{l}282 \\
294 \\
321 \\
323 \\
330 \\
311\end{array}$ & $\begin{array}{l}1977 \\
1977 \\
1978 \\
1978 \\
1978 \\
1977\end{array}$ & $\begin{array}{l}\text { Nov. } \\
\text { Dez. } \\
\text { Jan. } \\
\text { Febr. } \\
\text { Jan. } \\
\text { Nov. }\end{array}$ & $\begin{array}{l}\text { Contreras } \\
\text { El Aibe } \\
\text { Vta. de la B. } \\
\text { Los Cardozos } \\
\text { Simbol } \\
\text { San Isidro }\end{array}$ & $\begin{array}{l}\text { Hauptbezirk } \\
\text { Banda } \\
\text { Hauptbezirk } \\
\text { Hauptbezirk } \\
\text { Silipica } \\
\text { Capital }\end{array}$ & $\begin{array}{l}5,20 \\
5,42 \\
5,55 \\
5,80 \\
5,90 \\
5,96\end{array}$ \\
\hline $\begin{array}{l}314 \\
270 \\
317 \\
291 \\
331 \\
303 \\
322\end{array}$ & $\begin{array}{l}1978 \\
1978 \\
1977 \\
1978 \\
1978 \\
1977 \\
1978\end{array}$ & $\begin{array}{l}\text { Jan. } \\
\text { Jan. } \\
\text { Nov. } \\
\text { Febr. } \\
\text { Febr. } \\
\text { Nov. } \\
\text { März }\end{array}$ & $\begin{array}{l}\text { Los Romanos } \\
\text { Arraga } \\
\text { Tramo } 26 \\
\text { Antajé } \\
\text { Fernandez } \\
\text { El Mojón } \\
\text { Tala Pozo }\end{array}$ & $\begin{array}{l}\text { Banda } \\
\text { Silipica } \\
\text { Banda } \\
\text { Banda } \\
\text { Robles } \\
\text { Hauptbezirk } \\
\text { Hauptbezirk }\end{array}$ & $\begin{array}{l}6,03 \\
6,27 \\
6,39 \\
6,43 \\
6,50 \\
6,67 \\
6,96\end{array}$ \\
\hline 333 & 1978 & Febr. & Est. Ing. Ezc. & Hauptbezirk & 7,19 \\
\hline 319 & 1978 & Febr. & San Vincente & Silipica & 8,04 \\
\hline 305 & 1977 & Dez. & P. Aguirre & Hauptbezirk & 9,42 \\
\hline 307 & 1977 & Dez. & Est. Loreto & Loreto & 10,15 \\
\hline 310 & 1977 & Dez. & Añatuya & Gral. Taboada & 12,66 \\
\hline 268 & 1978 & Jan. & Silipica & Silipica & 13,80 \\
\hline
\end{tabular}


Die Messung der elektrischen Leitfähigkeit gestattet es, kleine Änderungen des Elektrolytgehalts schnell und sicher festzustellen. Sie ist daher handlicher als die Bestimmung des Aschegehalts, die etwa den gleichen Aussagewert hat.

Eingegangen im August 1979.

Reçu pour publication en aồt 1979.

\section{DANK}

Die Übersetzung aus dem Castellano ins Deutsche wurde freundlicherweise von Frau Carmen Cabezas Meyer durchgeführt.

\section{RÉSUMÉ}

On a récolté 36 échantillons de miel dans la province de Santiago den Estero dans le nord de l'Argentine et utilisé pour les mesures des solutions de miel à $20 \%$ (rapporté au poids sec) à la température de $20^{\circ} \mathrm{C}$. Les valeurs de la conductibilité électrique varient entre 1,73 et $13,80 \cdot 10^{-4} \mathrm{~S} \cdot \mathrm{cm}{ }^{~}{ }^{\prime}$. Ceci correspond au domaine de variation relevé pour les miels européens.

\section{LITERATUR}

BRITton H. S., 1934. - Conductometric Analysis. Chapman and Hall, London.

Davies C.W., 1933. - The Conductivity of Solutions, 2. Auflage, Chapman and Hall, London.

Delahay P., 1967. - Análisis Instrumental, 2. Auflage, Paraninfe, Madrid.

Vorwoнl G., 1964 a. - Die Messung der elektrischen Leitfähigkeit des Honigs und die Verwendung der Messwerte zur Sortendiagnose und zum Nachweis von Verfälschungen mit Zuckerfütterungshonigen. Z. Bienenforschung 7, 37-47.

Vorwoнl G., 1964 b. - Die Beziehungen zwischen der elektrischen Leitfähigkeit der Honige und ihrer trachtmässigen Herkunft. Ann. A beille 7, 301-309. 